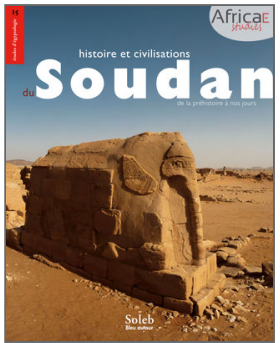

Histoire et civilisation du Soudan

De la préhistoire à nos jours

\title{
Noureddine Satti
}

\section{Odile Nicoloso}

DOI : 10.4000/books.africae. 2947 Éditeur : Africae, Soleb, Bleu autour Lieu d'édition : Paris, Khartoum Année d'édition : 2017

Date de mise en ligne : 17 janvier 2022

Collection : Africae Studies

EAN électronique : 9782493207074

\section{OpenEdition \\ Books}

http://books.openedition.org

Référence électronique

NICOLOSO, Odile. Noureddine Satti In : Histoire et civilisation du Soudan : De la préhistoire à nos jours [en ligne]. Paris, Khartoum : Africae, 2017 (généré le 28 janvier 2022). Disponible sur Internet : <http:// books.openedition.org/africae/2947>. ISBN : 9782493207074. DOI : https://doi.org/10.4000/ books.africae. 2947. 

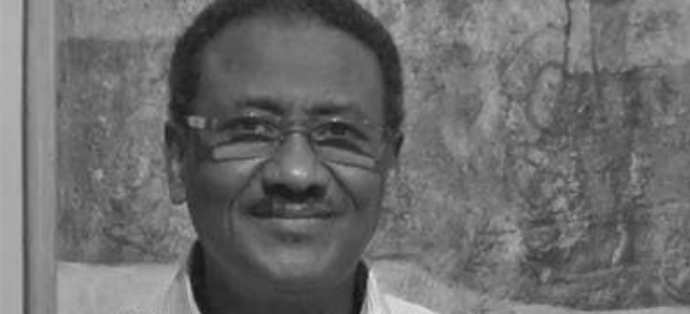

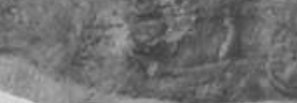
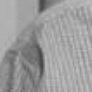

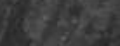
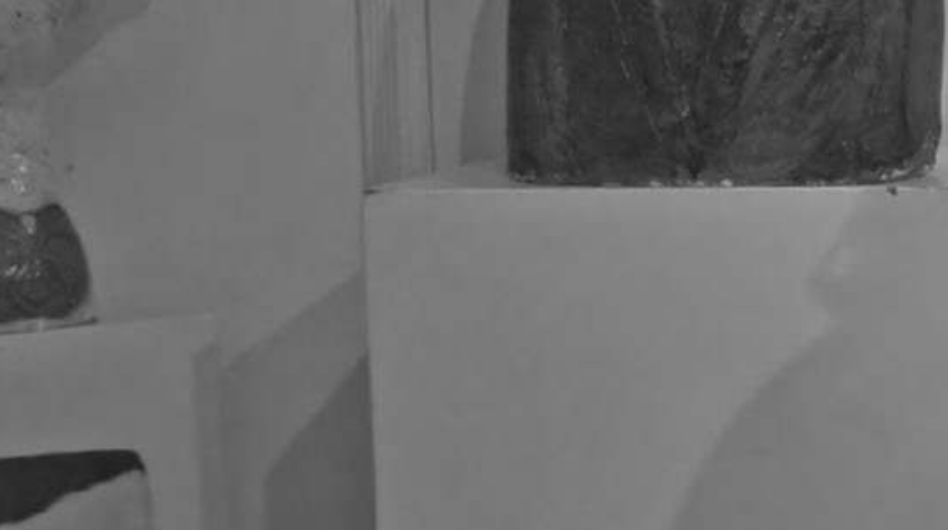


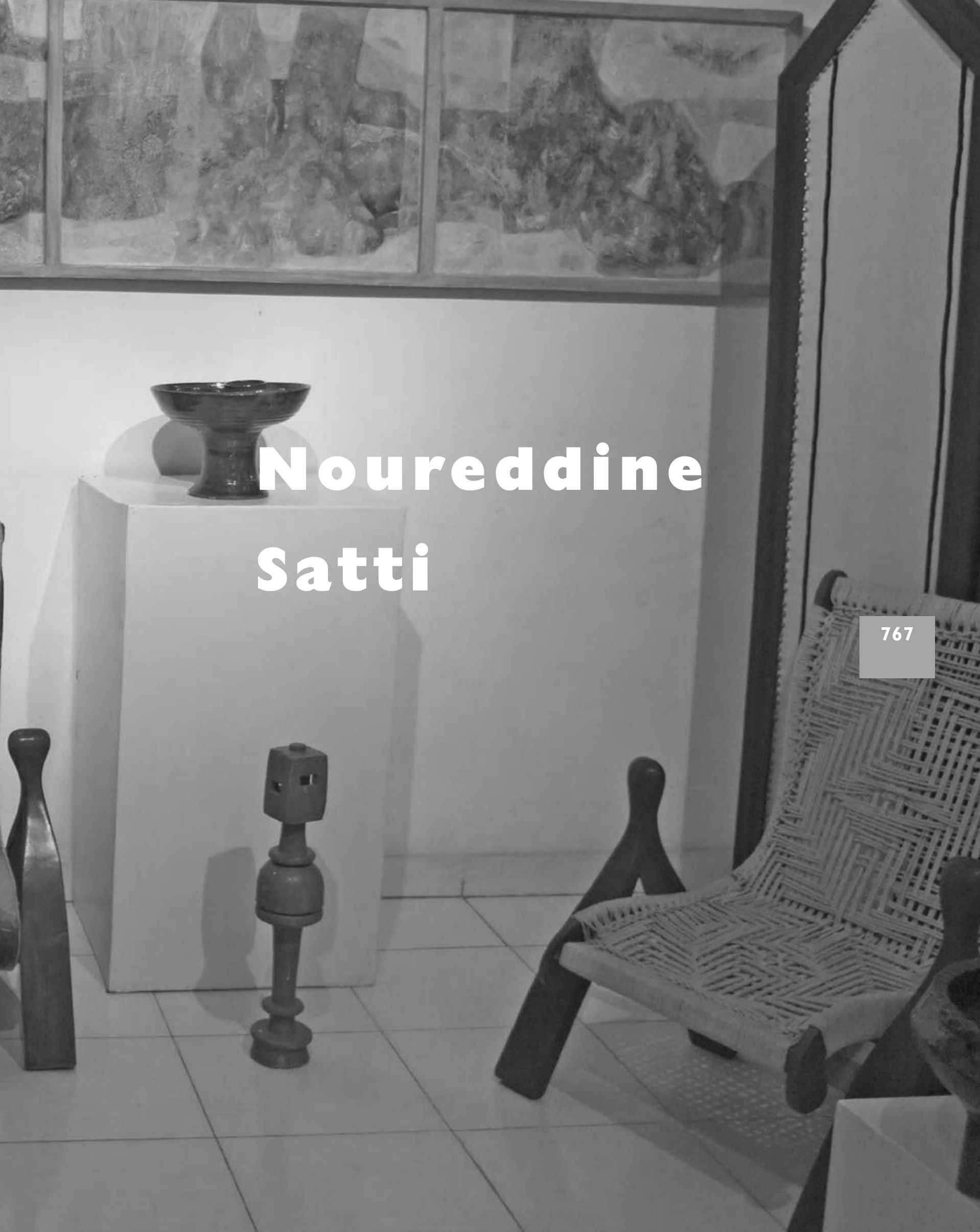


le Soudan

\section{Islamisme et politique au Soudan}

aujourd'hui dans la paix et la réconciliation.
Noureddine Satti est d'origine nubienne et sa famille émigra à Omdurman dans la deuxième moitié du XIX ${ }^{e}$ siècle. Il a fait ses études à Lyon et est devenu professeur de français à l'université de Khartoum (1970). Diplomate, il a exercé la charge d'ambassadeur à l'Unesco et à Paris de 1992 à 1996. Il a également été en poste au Burundi et s’intéressa au conflit entre Hutus et Tutsis. Dans son itinéraire, sa préférence va à la diplomatie culturelle, pour laquelle il a pu œuvrer lors de l'exposition «Royaumes sur le Nil», organisée à l'institut du Monde arabe à Paris en 1991. Il a écrit en 1974 un roman, non encore publié, intitulé Nil Bleu Nil Blanc, où il développe la symbolique du fleuve - patrimoine historique et richesse - qui se réunit à Khartoum

Il aimerait donner une meilleure image du Soudan — qui est surtout connu comme un pays en conflit permanent —, le réconcilier avec lui-même et l'ouvrir au monde. Car ce pays multiculturel (africain, arabe, musulman, chrétien) a pour dénominateur commun la générosité, la tolérance, l'ouverture d'esprit et l'hospitalité. Ces valeurs ont été bafouées par certains politiques extrémistes qui ont instauré la méfiance, la compétition et la corruption. Noureddine Satti voudrait populariser le concept de soudanité, qui serait partage avec l'autre, citoyenneté, égalité, rassemblement

Depuis la formation du Soudan en tant qu'État au début du XIX siècle, l'islam constitue un élément fondamental de son évolution politique et sociale et de ses relations avec ses voisins et avec les puissances internationales. Le sursaut mahdiste contre l'occupation turco-ottomane, accompagné de la création d'un État indépendant (1884-1898), n'était autre qu'un acte de libération nationale animé par de profonds sentiments religieux dont l'objectif immédiat était de débarrasser le pays du joug de l'occupation.

Mais la révolution mahdiste visait également l'objectif de « rétablir la justice dans le monde après qu'il fut rempli d'injustice». Ce faisant, la mahdiyya défiait les puissances régionales - notamment l'Égypte et l'Éthiopie - et mondiales, en l'occurrence la Turquie et la Grande Bretagne. Finalement, la chute de l'État mahdiste fut provoquée par la conjonction de facteurs internes et externes, dont les querelles intestines, la mauvaise gestion des affaires de l'État, la prévalence des approches militaro-sécuritaires pour résoudre les problèmes et la confusion des objectifs internes et externes: on a voulu réformer le monde sans en avoir les moyens matériels, politiques ou diplomatiques. 
Contre vents et marées, ce schéma ne cesse de se reproduire dans le domaine politique soudanais. En effet, depuis son indépendance en janvier 1956, le Soudan ne réussit pas à concilier les diversités multiples de la société soudanaise avec une approche de gouvernance unilatérale et unidimensionnelle. Cela équivaut à vouloir faire entrer un chameau dans le chas d'une aiguille, comme il est dit dans le Coran.

En effet, la question de l'identité a été, dès le début, au centre du débat: identité ethnique, religieuse et linguistique. Tout au long de ces décennies, les élites du pouvoir central et celles des régions dites marginalisées se sont disputées, sans pour autant arriver à une formule consensuelle pour la gouvernance politique et socio-économique du pays. Cette situation perdure encore aujourd'hui. Mais, de plus en plus, l'islamisme émerge comme un phénomène politique plutôt que religieux.

Dans l'esprit des pères fondateurs de l'islamisme "moderne» incarné dans le mouvement des Frères musulmans en Égypte, au Soudan ou ailleurs —, la renaissance islamique doit passer obligatoirement par le démantèlement de l'État laïc et la mise en place de structures étatiques fondées sur les préceptes islamiques, parfois puisés dans des références historiques n'ayant pas de fondements solides et ne répondant pas aux exigences du monde contemporain.

Le modèle islamiste se veut un système global qui définit et contrôle la vie des individus et de la communauté; l'islam, c'est deenun wa duniya, c'est-à-dire à la fois une religion et un mode de vie. L'islam doit donc régler tous les aspects de la vie des croyants, jusqu'aux détails les plus minutieux, par exemple édicter s'ils doivent porter une barbe, écourter leur jallabah ou manger de la main droite.

Mais puisqu'il s'agit de traiter ici des aspects politiques de l'islamisme soudanais, nous nous efforcerons de retracer son cheminement depuis l'accès du pays à l'indépendance, en 1956, jusqu'à nos jours.

Le Soudan a conquis son indépendance grâce au militantisme de l'élite constituée suite à l'introduction de l'éducation moderne au début du $\mathrm{xx}^{\mathrm{e}}$ siècle par les colonisateurs britanniques. Dès les années trente, les fruits politiques de cette éducation commencent à apparaître. Le mouvement des lettrés, appelé les khirrejeen (les diplômés), s'est constitué en tant que mouvement de résistance sociale et intellectuelle pacifique à la colonisation mais s'est vite transformé en un mouvement politique, avec la création de deux partis politiques: le parti des ashigaa (c'est-à-dire les "frères») qui prônait l'union avec l'Égypte, s'est vite scindé en deux branches, le Parti nationaliste unioniste et le Parti démocratique du peuple (PDP), qui ont par la suite de nouveau fait un. Déjà, cette scission avait pour cause la relation entre la religion et la politique: le PDP devenait officieusement le parti 
de la confrérie Khatmiyya, tandis que l'Al-Hizb al-Watani Alittihadi optait pour la séparation entre la politique et la religion, tout en gardant un lien minimal avec la confrérie.

L'autre parti créé à cette époque fut l'Umma, émanant directement de la confrérie des Ansar, sous l'égide de la famille du grand combattant du XIx ${ }^{e}$ siècle Muhammad Ahmad ibn Abd Allah al-Mahdi. Ce parti préconisait l'indépendance du Soudan, contrairement à son rival, le Parti nationaliste unioniste, qui avait opté, dans un premier temps au moins, pour une union avec l'Égypte.

Avant l'indépendance, le Soudan a donc connu l'interpénétration entre la politique et le fait religieux. Les deux grandes confréries religieuses, les Khatmiyya et les Ansar, jouaient déjà un rôle prépondérant sur l'échiquier politique. L'élite politique avait du mal à se dégager de l'emprise des chefs religieux. Cela dit, ceux-ci se gardaient bien d'imposer un agenda religieux strict, car ils prônaient la tolérance et prêchaient une transformation graduelle de la société compatible avec ses capacités. Cette version "soft» de l'islam correspondait bien à la société rurale et pastorale qui pratiquait un Islam imbibé d'esprit de convivialité et de solidarité naturelle. Cette version s'adaptait aussi avec le manque d'éducation et la survivance d'un certain féodalisme: le chef d'une confrérie religieuse avait un pouvoir quasi absolu sur ses adeptes, y compris dans le domaine spirituel. Ce rapport d'allégeance absolue se transposait dans le domaine politique et social et créait une certaine harmonie dans la communauté, car ni l'un ni les autres ne se posaient trop de questions sur cette relation. Du fait de la modernisation progressive de la société et l'émergence de nouvelles classes sociales plus éclairées et émancipées, du fait aussi des influences venues de l'extérieur, notamment de l'Égypte et de l'Arabie Saoudite, la donne a commencé à changer. Mais c'est l'influence de l'Arabie Saoudite qui fut prépondérante, particulièrement à partir des années quatre-vingt.

L'islamisme soudanais commence alors à changer de visage. Les deux grands partis populaires et les deux mouvements politicoreligieux, les Khatmiyya et les Ansar, furent considérablement affaiblis sous le régime du président Nimeiry. Le mouvement des Frères musulmans s'est graduellement transformé, sous l'égide de Hassan al-Tourabi : mouvement de l'élite urbaine à l'origine, il s'est mué en un vaste conglomérat d'individus. L'émigration vers l'Arabie Saoudite et les pays du Golfe a progressivement changé la donne économique et sociale, donnant naissance à une nouvelle classe moyenne liée aux pays du Golfe par des intérêts matériels et des attaches spirituelles et culturelles. Ce changement intervint au moment même où l'on assistait à l'affaiblissement 
des attaches culturelles et académiques avec les pays occidentaux, partenaires traditionnels du Soudan, et où l'on commençait à remettre en question le modèle occidental de développement. Cette aspiration émancipatrice a été saisie par le régime du président Nimeiry qui souffrait d'un certain essoufflement; lancer des appels à la shari'a était aussi pour lui une opportunité de mater le mouvement de protestation qui risquait de menacer son pouvoir. Ainsi promulgua-t-il en 1983 les «Lois de septembre» qu'il utilisa comme un outil punitif contre les opposants au régime, en mettant l'accent sur les hudud (châtiments corporels) et en vidant les enseignements de l'islam de tout leur contenu de tolérance, d'humanisme et de bienveillance. Les mauvais débuts ont de mauvaises fins: le renversement de Nimeiry peut être attribué, dans une certaine mesure, aux excès de cette période.

La proclamation de la shari'a par le maréchal Nimeiry a accru la polarisation de la classe politique soudanaise entre un camp libéral et un camp islamiste. Mais cette proclamation a constitué un piège impossible à déjouer : même si l'application de la shari'a telle que pratiquée par Nimeiry n'était qu'une mascarade et une parodie de la vraie shari'a, personne n'osait annuler ou suspendre son application de crainte de contrarier les enseignements de l'islam.

Même si l'annulation des Lois de septembre fut l'une des revendications du soulèvement populaire de 1985 , qui mit fin au règne du maréchal Nimeiry, ni le gouvernement de transition du général Swar el-Dahab ni celui démocratiquement élu de Sadiq al-Mahdi ne furent en mesure d'annuler ces lois anti-démocratiques et répressives qui n'avaient de la shari'a que le nom.

Ainsi, les lois « islamiques» devaient rester... et celui qui fut l'éminence grise et conseiller juridique du président Nimeiry, le docteur Hassan al-Tourabi, persista et signa, déjà en jouant un rôle déterminant dans le soulèvement populaire qui renversa le régime. Un gouvernement d'union nationale fut alors mis en place sous l'égide de Sadiq al-Mahdi, un Premier ministre aussi indécis qu'inefficace qui ne devait gouverner que trois ans... pendant lesquels le docteur al-Tourabi (dont le parti des Frères musulmans participait au gouvernement) s'appliquait à ce que le pays soit totalement ingouvernable. Jusqu'au coup d'État de juin 1989 qui a amené au pouvoir le régime islamiste du colonel Omar Hassan Ahmed el-Bechir.

La politique du nouveau régime islamiste se basait sur deux piliers: à l'intérieur, le tamkin, autrement dit la totale prise en main de tous les leviers de l'État et de la société, et, à l'extérieur, la politique des frontières ouvertes qui consistait à répandre l'idéologie islamiste et à soutenir la cause des mouvements islamistes et djihadistes partout dans le monde. 
À l'intérieur, ce fut la chasse aux sorcières qui visait les adversaires politiques et tous ceux qui avaient montré, de près ou de loin, une opposition quelconque aux thèses des Frères musulmans. Ceux qui ont été licenciés furent rapidement remplacés par des personnes fidèles aux thèses des islamistes; elles furent recrutées moins pour leur compétence que pour leur la loyauté politique et idéologique. L'application rigoureuse de cette politique discriminatoire a vidé le pays de ses cadres les plus qualifiés et causé l'effondrement de la fonction publique et des institutions de l'État.

Sur le plan extérieur, la politique dite des frontières ouvertes a fait le plus grand mal. Le Congrès populaire et islamiste — présidé par le docteur Hassan al-Tourabi — réunissait des islamistes de tous bords, y compris des éléments qui faisaient partie des groupes militant contre les intérêts des pays occidentaux dans plusieurs parties du monde, notamment en Afghanistan, en Afrique et au Moyen Orient. Oussama Ben Laden, avant de se réfugier dans les montagnes de Bora Bora, a été l'hôte des autorités de Khartoum

Et c'est la stabilité et l'unité du Soudan qui devaient en souffrir. La grande erreur du docteur Tourabi, du général el-Bechir et de leurs collègues, c'est de n'avoir pas su prendre en compte les évolutions survenues. Le hasard a voulu que la "Révolution du Salut", l'inqaz, survienne au Soudan en même temps que la chute du mur de Berlin qui entraîna la rupture de l'équilibre entre le monde occidental et le bloc soviétique, puis l'effondrement de ce dernier. Les appels au renouveau islamique se mêlaient à la volonté de se débarrasser et du joug occidental et de la tyrannie soviétique qui venait d'être vaincue en Afghanistan. Les mujahideen islamistes, qui avaient enregistré une victoire en terre afghane, en cherchaient d'autres en terre d'islam ou partout ailleurs. Le combat contre les forces anti-islamistes ou supposées telles engloba les musulmans modérés... mais considérés comme des laïcs ou des libéraux par les islamistes purs et durs.

L'islamisme soudanais ne fait pas exception: son objectif ultime est l'islamisation de la société par des moyens musclés qui vont à l'encontre des principes les plus élémentaires de respect du droit d'expression et de liberté religieuse, politique et sociale. Tandis que toutes les composantes de l'islamisme soudanais s'accordent sur les fondements de base d'un État islamiste (respect des huduud, port du hijab pour les femmes, application des lois islamiques en matière d'état civil et dans le système bancaire, interdiction de l'alcool et stricte observance des furoud en ce qui concerne les prières, le jeûne et les autres fondements de l'islam), de nouvelles variantes de l'islam wahabite et djihadiste ont fait leur irruption sur la scène ces dernières années. En effet, on assiste depuis les années soixante-dix à 
une radicalisation progressive des mouvements islamistes. Les spécificités locales se sont effacées au profit de préceptes purs et durs provenant d'ailleurs. Au Soudan, cette radicalisation s'est traduite par l'affaiblissement du mouvement soufi et le dépassement des confréries religieuses traditionnelles par des mouvements d'inspiration moderniste, comme celui des Frères musulmans, ou par des mouvements encore plus radicaux tels que les Ansar Assunna, ou d'autres mouvements d'inspiration wahhabite ou salafiste, comme le Parti de Tahrir pour qui les sociétés musulmanes ne peuvent être considérées comme telles tant qu'elles n’ont pas appliqué la shari'a et instauré le califat.

Au Soudan comme dans d'autres pays musulmans, l'islam soi-disant politique n'a pas pu répondre aux grandes interrogations du moment: comment concilier démocratie et shura? comment concilier les instruments internationaux des droits de l'Homme avec le droit et la jurisprudence islamiques? comment concilier les exigences de la shari'a et de la loi islamique avec la liberté individuelle, politique et sociale prônée par les sociétés modernes? comment concilier le système financier prôné par l'islam et le système international financier? en bref, comment concilier islam et modernité?

La démarche qui consiste en un refus total de la modernité a donné naissance à des mouvements radicaux, tels qu'Al-Qaïda, Boko Haram, Chabab et, dernièrement, Ansar Eddine au Mali. Ces mouvements sont nés de sentiments de frustration d'une fraction grandissante de la jeunesse musulmane face à l'injustice, à l'abandon et à la corruption aux niveaux local et international. L'échec des modèles de développement importés de l'Occident à répondre à leurs aspirations légitimes et la corruption des élites au pouvoir sont autant de facteurs alimentant ces mouvements de refus politique et social. De profondes réformes aux niveaux national et global s'imposent pour contrecarrer ces mouvements. Une réponse uniquement militaire et sécuritaire risque de compliquer davantage ces problèmes. Des approches politiques, socio-économiques et culturelles sont requises pour venir à bout de ces mouvements.

Au Soudan, la résolution des problèmes complexes auxquels le pays est confronté doit passer obligatoirement par un dialogue ouvert, franc et sincère entre toutes les composantes politiques et sociales de la société. Ce dialogue doit traiter les questions constitutionnelles, politiques, socio-économiques et culturelles pour répondre aux attentes des populations, surtout dans les régions marginalisées qui n’ont que trop souffert et qui ont droit à une vie plus décente. Cette résolution doit consacrer le fait que l'unité dans la diversité pourrait être le meilleur atout de l'islam... au Soudan, comme partout ailleurs $=\$\rangle$ 
La question identitaire est au centre des conflits qui se sont déroulés au Soudan depuis son indépendance en 1956. Avant la sécession du Sud Soudan, il y avait au Soudan deux grandes identités qui se faisaient la guerre: une qui se revendiquait de l'arabo-islamisme et une autre du négroafricanisme. Ces deux identités, transformées en idéologies contradictoires, n'ont jamais réussi à trouver un terrain d'entente ou un modus vivendi qui assurerait à ces deux grandes composantes de la société soudanaise une coexistence pacifique. La persistance d'une idéologie de l'exclusion et de la marginalisation a été l'une des causes principales de la sécession du Sud Soudan. Cet échec à maintenir l'unité du pays est surtout imputable à un autre échec, qui est d'avoir escamoté le débat autour de la question identitaire en voulant lui donner une explication unilatérale assurant la mainmise d'une idéologie islamiste et arabisante sur les populations du Sud Soudan qui se voyaient considérées comme de deuxième degré. Même la solution "d'un État, deux systèmes », préconisée par l'accord de Naivasha, n’a pas pu résoudre le problème du fait de la méfiance et du manque de confiance qui s'étaient installés entre les décideurs politiques des deux bords.

Mais la question aurait pu être réglée avec un peu de bonne volonté: il s'agissait de reconnaître le droit de tout un chacun de pratiquer sa culture, sa religion ou d'user de sa langue ou de ses traditions, dans le cadre d'une société démocratique, plurielle et réconciliée avec elle-même. Quoi de plus simple? Mais au Soudan, la simplicité, surtout quand il s'agit des choses de la culture ou de l'identité, n'est pas monnaie courante. Les théoriciens de l'identité représentant les deux écoles de pensée se sont coalisés et ont créé des lobbies, des groupes d'intérêts et même, parfois, des mouvements armés, souvent en utilisant les moyens de l'État, pour chercher à imposer leurs points de vue, au nom de la religion, de la culture, de la langue, de la région ou de l'ethnie. Le débat, souvent musclé, sur la question identitaire est devenu la pierre d'achoppement de toute tentative de dialogue ou de réconciliation nationale. Même aujourd'hui il est en passe de devenir une cause du démantèlement du projet de construction nationale.

Il est indéniable que l'identité soudanaise, s'il y en a une, est une identité diverse, multiforme, multilinguistique, multiethnique et multiraciale. D'aucuns prétendent, à tort, que, suite à la sécession du Sud Soudan, ce qui reste du pays est devenu homogène et que le Soudan jouit dorénavant d'une identité unique arabo-islamique. Le fait que la majorité des Soudanais soient des musulmans et qu'ils utilisent la langue 
arabe comme moyen de communication ne doit pas cacher le fait qu'ils ne sont pas ethniquement des Arabes, ni celui que, bien que musulmans, ils refusent que l'islam — ou, plutôt, l'islamisme - soit invoqué pour confisquer leurs droits les plus élémentaires. L'idéologie islamiste et arabisante telle que prônée et appliquée au Soudan mène à la hiérarchisation de la société sur des bases ethniques, fait croître les revendications de marginalisation des «autres» et incite des franges grandissantes des populations des régions périphériques à contester l'ordre établi par tous les moyens à leur disposition, y compris l'action militaire. Ainsi, la question identitaire, jumelée avec d'autres revendications politiques, économiques ou sociales, est en passe de constituer une véritable menace pour la cohésion et l'unité du pays.

Pour remédier à ce mal profond qui gangrène la société et l'État, il faut changer de cap, autrement dit reconnaître la diversité de la société soudanaise et le fait que cette diversité, si elle est bien gérée, pourrait être source de force et de richesse. Une nouvelle approche est requise qui mettrait à l'aise toutes les composantes de la société, dans leurs diversités ethniques, linguistiques, religieuses, politiques et sociales. Cette approche ne peut être que démocratique, tolérante et transparente. L'application judicieuse des principes cardinaux de l'équité et de la justice sociale aboutirait à ce que tous les Soudanais soient égaux, quelles que soient leur langue, leur religion ou leur appartenance ethnique. C'est là le fondement et la pierre angulaire de la construction de la société soudanaise de demain. C'est là aussi où le débat, pour ne pas dire la bataille, en vue de la nouvelle constitution et de la transformation démocratique revêt toute son importance. Il faudrait créer les conditions propices pour que ce débat se déroule sereinement. Un débat libre et transparent est nécessaire pour mettre fin aux guerres des identités ethniques et régionales qui menacent la cohésion sociale et l'unité nationale. C'est ainsi que la question identitaire, perçue aujourd'hui comme source de conflit et de discorde, pourrait devenir un facteur de paix et de cohésion sociale

\section{Noureddine Satti}

Khartoum, 15 mai 2013 